\title{
Quasar absorption line studies of galaxies and the
}

intergalactic medium at $\mathrm{Z}>1.5$

Lisa J. Storrie-Lombardi

Citation: AIP Conference Proceedings 470, 79 (1999); doi: 10.1063/1.58638

View online: http://dx.doi.org/10.1063/1.58638

View Table of Contents: http://aip.scitation.org/toc/apc/470/1

Published by the American Institute of Physics 


\title{
Quasar Absorption Line Studies of Galaxies and the Intergalactic Medium at $\mathrm{z}>\mathbf{1 . 5}$
}

\author{
Lisa J. Storrie-Lombardi \\ Carnegie Observatories, Pasadena, CA 91101, lisa@ociw.edu
}

\begin{abstract}
The title of this article could of course encompass an entire meeting. I will focus my comments on reviewing of what we know about the most numerous absorption lines, the neutral hydrogen absorbers, and their evolution with redshift. This field of study has undergone a renaissance in last few years driven by observations with the Hubble Space Telescope of low redshift quasar absorption lines, observations of high redshift absorbers with the HIRES instrument on Keck, and cosmological modeling that allows us to make detailed comparisons of lines of sight through simulated universes.
\end{abstract}

\section{INTRODUCTION}

Bright high redshift quasars are particularly valuable as probes of the intervening gas clouds and galaxies superimposed on their spectra in absorption. The galaxies that intercept their lines of sight provide samples selected by gas cross-section, without respect to their surface brightness, luminosity, or star formation rate. Though direct studies of high redshift galaxies are now possible, those selected by the absorption lines they produce in QSO spectra still provide the only means to study in detail their kinematic properties at high resolution. Optical spectroscopy from ground-based telescopes and ultraviolet spectroscopy from space have provided us with a wealth of information about the ionized intergalactic medium and neutral gas and metals in galaxies from very low redshifts out to nearly $z=5$. Recent advances in cosmological hydrodynamic simulations have provided us real insight into the physical properties of the structures that produce the Ly $\alpha$ forest lines.

In the following sections I briefly review the definitions of the different classes of HI absorption systems, and discuss their evolution in number density with redshift, determinations of their metal abundances, and the interpretations of these results.

CP470, After the Dark Ages: When Galaxies were Young (the Universe at $2<z<5$ ), edited by Stephen S. Holt and Eric P. Smith

(C) 1999 The American Institute of Physics 1-56396-855-X/99/\$15.00 


\section{TAXONOMY OF NEUTRAL HYDROGEN ABSORBERS}

Neutral hydrogen absorption can be detected over a staggering 10 orders of magnitude from the Ly $\alpha$ forest region with the weakest detectable lines having a column density $\mathrm{N}_{\mathrm{HI}} \sim 10^{12}$ atoms $\mathrm{cm}^{-2}$, up to the damped Ly $\alpha$ absorbers with $\mathrm{N}_{\mathrm{HI}} \sim 10^{21}$. The rich zoo of these absorbers, in addition to those produced by heavier elements such as carbon, silicon, oxygen, and magnesium, are illuminated along a quasar line-of-sight, leaving their imprint as absorption in the quasar continuum. HI absorbers are typically divided into three classes based on their column density.

- Ly $\alpha$ Forest: $\left(\mathrm{N}_{\mathrm{HI}}=10^{12}\right.$ to $10^{16}$ atoms $\left.\mathrm{cm}^{-2}\right)$ Figure 1 shows a high redshift quasar spectrum. We see the ultraviolet emission lines Ly $\alpha(1216 \AA)$ and CIV $(1549 \AA)$ redshifted to $\sim 6600 \AA$ and $8300 \AA$ respectively. All of the absorption structure blueward of the Ly $\alpha$ emission is real, and almost all the lines in this "forest" are neutral hydrogen. Studies of the forest yield a wealth of information about the intergalactic medium, and the background ionizing flux, and structures at high redshifts (see [1] for a complete review of the Ly $\alpha$ forest absorbers). These absorbers are characterized by low metal content, some have been shown to be associated with galaxies, and their number density increases rapidly with increasing redshift.

- Lyman-Limit Systems: $\left(\mathrm{N}_{\mathrm{HI}} \geq 1.6 \times 10^{17}\right.$ atoms $\left.\mathrm{cm}^{-2}\right)$ As we move to higher column densities, neutral hydrogen becomes optically thick to Lyman continuum radiation at wavelengths below $912 \AA$. The Lyman limit at $z=4.37$ can be seen in figure 1 at $4900 \AA$ where the flux sharply drops to zero. Lymanlimit systems provide a means of directly studying the evolution of galaxies over the redshift range $0.1<\mathrm{z}<5[2-5]$. They have been shown to be associated with normal galaxies for $z<1.6[6,7]$.

- Damped Ly $\alpha$ Systems: $\left(\mathrm{N}_{\mathrm{HI}} \geq 2 \times 10^{20}\right.$ atoms $\left.\mathrm{cm}^{-2}\right)$ The absorbers detected via the damped Ly $\alpha$ lines they produce show features consistent with an early phase of galactic evolution [8-11]. They are called "damped" because at these high column densities we see radiation damping wings in the line profile. A damped absorber can be seen at $5910 \AA$ in figure 1 at a redshift of $z=3.86$ [12]. The column densities of damped absorbers are comparable to what we see along a typical line of sight through our own galaxy. Though the lower column density forest lines are far more numerous, damped absorbers contain most of the neutral hydrogen in the Universe.

\section{NUMBER DENSITY EVOLUTION WITH REDSHIFT OF HI ABSORBERS}

Observations have long shown that as we move to higher redshifts, the number density of lower column density HI absorbers increase at a much faster rate than 


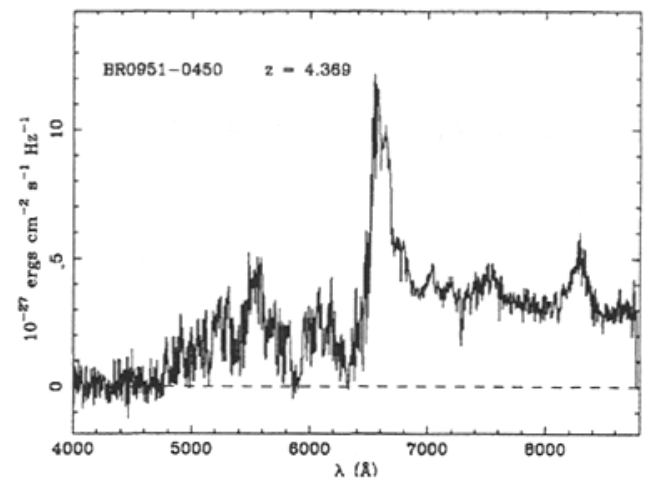

FIGURE 1. The spectrum of the quasar BRI0951-0450. The ultraviolet emission lines Ly $\alpha$ $(1216 \AA)$ and CIV $(1549 \AA)$ are redshifted to $\sim 6600 \AA$ and $8300 \AA$ respectively. All of the absorption structure blueward of the Lya emission is real, and almost all the lines in this "forest" are neutral hydrogen. The sharp drop in flux at $4900 \AA$ is due to a Lyman-limit system at $z=4.37$. A damped Ly $\alpha$ absorber at $z=3.86$ appears at $5910 \AA$ as the strong absorption trough. (Storrie-Lombardi et al. 1996)

for the higher column density systems, and it is clear that the Ly $\alpha$ forest as a whole evolves quite strongly with redshift.

In a standard Friedmann Universe for absorbers with cross-section $\pi R_{0}{ }^{2}$ and number density $\Phi_{0}$ per unit comoving volume

$$
N(z)=\Phi_{0} \pi R_{0}{ }^{2} c H_{0}^{-1}(1+z)\left(1+2 q_{0} z\right)^{-1 / 2} .
$$

It is customary to represent the number density as a power law of the form

$$
N(z)=N_{0}(1+z)^{\gamma},
$$

where $N_{0}=\Phi_{0} \pi R_{0}{ }^{2} c H_{0}^{-1}$. This yields $\gamma=1$ for $q_{0}=0$ and $\gamma=1 / 2$ for $q_{0}=1 / 2$ for the case of no evolution with redshift in the product of the number density and cross-section of the absorbers $[13,14]$.

For the Ly $\alpha$ forest lines $\left(\log \mathrm{N}_{\mathrm{HI}}<16\right) N(z)$ is calculated by either counting individual absorption lines [15-19] or by measuring the mean absorption, $\mathrm{D}_{\mathrm{A}}$, caused by the Ly $\alpha$ forest shortward of the QSO Ly $\alpha$ emission line [20-22]. Though there is a large scatter in the values of the exponent $\gamma$ determined by these different authors, ranging from 1.89 to 2.9 for $2<\mathrm{z}<4$ and with values of $\gamma>5$ for redshifts $z>4$, it is clear that for $\mathrm{z}>2$ the slope of the power law is steeper than would be expected for a non-evolving population, and probably steepens even further at redshifts $\mathrm{z}>4$. This is in contrast to the very flat slope $(\gamma=0.16 \pm 0.16)$ of the power law measured for redshifts $z<1.5$ [4] from the HST QSO Absorption Line Key Project data. With their complete data set they detect the steepening of the power law at $z \approx 1.7$, which matches well onto the data points determined 


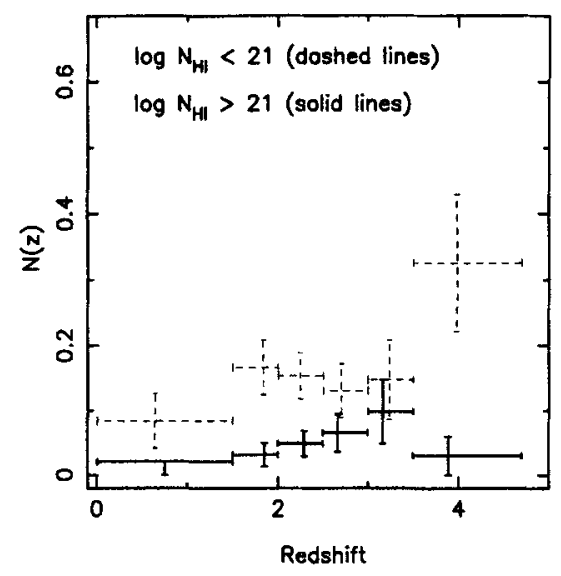

FIGURE 2. The number density per unit redshift, N(z), for damped Ly $\alpha$ absorbers is plotted versus redshift. Differential evolution with column density is evident. The higher column density absorbers $\left(\log N_{\mathrm{HI}}>21\right.$, plotted as solid lines) disappear at a faster rate from $\mathrm{z} \approx 3$ to $\mathrm{z}=0$ than the lower column density damped systems $\left(\log N_{\mathbf{H I}}<21\right.$, plotted as dashed lines). A paucity of very high column density absorbers is evident at $\mathrm{z}>4$ (Storrie-Lombardi \& Wolfe, in preparation).

from ground-based observations. Recent many-body simulations tracing collisionless particles in cold dark matter scenarios ( [24], and references therein) reproduce the observations well. By modeling the evolution of the UV background radiation, as well as allowing for shock heating, the simulations indicate that the unshocked material is found primarily in underdense regions while the shocked material is found in condensing regions. The unshocked population evolves more rapidly with increasing redshift, and dominates the absorbers at high redshift. Conversely, the shocked population has a flat evolution and dominates at low redshift.

The Lyman Limit systems $\left(\log \mathrm{N}_{\mathrm{HI}}>17\right.$ ), on the other hand, are consistent with no evolution with redshift in the product of their number density and crosssection $[2,4,5]$ or mild evolution (for an $\Omega=1, \lambda=0$ universe), with $\gamma=1.55 \pm 0.3$ for redshifts $0.008<\mathrm{z}<4.7$ [4]. A two power law fit, with $\gamma \approx 1$ for $\mathrm{z}<1$ and $\gamma \approx 2.8$ for $z>1$ is also consistent with the data but is not required. The damped absorbers $\left(\log \mathrm{N}_{\mathrm{HI}}>20.2\right)$ yield a similar value for the exponent $\gamma(1.11 \pm 0.40)$ but show differential evolution with column density $[8,25,26]$. The highest column density damped systems $\left(\log N_{\mathrm{HI}}>21\right)$ disappear at a faster rate from $\mathrm{z} \approx 3$ to $\mathrm{z}=0$ than the lower column density subset and there is a paucity of very high column density absorbers at $\mathrm{z}>4$ (figure 2 ). 


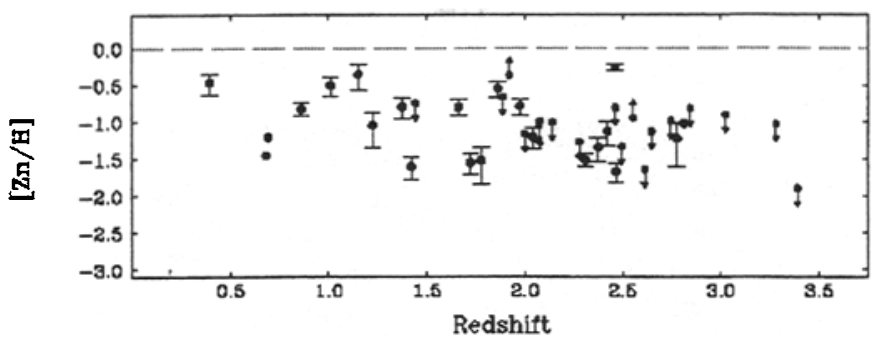

FIGURE 3. The $\mathrm{Zn} / \mathrm{H}$ abundance for a large sample of damped Ly $\alpha$ absorbers is plotted versus redshift. The damped Ly $\alpha$ systems are metal poor at all redshifts, with a mean abundance of $\langle Z n / H\rangle=-1.13 \pm 0.38$, about $1 / 13$ of solar. There is a large scatter in metallicity at a given redshift and the measured metallicities are lower at higher redshifts (Pettini et al. 1999).

\section{MEASUREMENTS OF METAL ABUNDANCES IN HI ABSORBERS}

One of the paradigm shifts that has occurred in QSO absorption studies in recent years is the detection of metals associated with the Ly $\alpha$ forest lines, which were initially thought to be primordial HI clouds. The high resolution/high signal-tonoise spectra that can be obtained with the HIRES instrument [27] on the Keck telescope have made this possible. For HI absorbers with column densities $\log \mathrm{N}_{\mathrm{HI}}$ $\approx 15$, most show associated CIV absorption, and for those with $\mathrm{N}_{\mathrm{HI}}>3 \times 10^{14}$, about 50 percent show associated CIV lines, with a typical metallicity $\mathrm{Z} / \mathrm{Z}_{\odot}=10^{-2}$ $[28,29]$.

Substantial progress has also been made in measuring metal abundances in the damped Ly $\alpha$ absorbers. A large amount of time on $4 \mathrm{~m}$ telescopes $[30,11,31]$ and the Keck 1 telescope $[32,10]$ has been applied to this project. The picture that emerges is:

1. Damped Ly $\alpha$ systems are metal poor at all redshifts, with a mean abundance of $\langle Z n / H\rangle=-1.13 \pm 0.38$, about $1 / 13$ of solar.

2. There is a large scatter in metallicity at a given redshift.

3. The measured metallicities are lower at higher redshifts.

4. The damped system abundances don't match the disk star abundances.

5 . The velocity profiles from high resolution spectra of low ionization metal lines have multiple, narrow components and are asymmetric in that the component with the strongest absorption tends to lie at one edge of the profile.

Points 1-3 are illustrated in figure 3 from Pettini et al. (1999) [31]. There is still considerable controversy though about exactly how to interpret the results from observations of damped absorbers. 
Prochaska \& Wolfe (1997a, 1997b) [33,34] have argued that the observationally determined velocity profiles are reflective of rapidly rotating, cold disks. The low ionization metal lines observed in damped systems accurately trace the velocity fields of the neutral gas dominating the baryonic content of the absorbers. Haehnelt, Steinmetz \& Rauch (1998) [35] propose a model that reproduces the observed velocity profiles that is consistent with smaller clumps or galactic building blocks, not large disks. Pettini et al. (1997) [11] suggest that damped Ly $\alpha$ systems are drawn from a varied population of galaxies of different morphological types and at different stages of chemical evolution, supporting the idea of a protracted epoch of galaxy formation. When our Galaxy's metal enrichment was at levels typical of damped systems, its kinematics were closer to those of the halo and bulge than a rotationally supported disk. Lu et al. (1996) [10] also found that the chemical evolution history of damped systems is more consistent with the spheroidal component of galaxies or dwarf galaxies.

The interpretation of the elemental ratios measured in damped systems is also the subject of debate. They have been interpreted to be consistent with mild dust depletion, dust depletion combined with supernovae type II enrichment, or supernovae type II enrichment alone. We also do not have definitive evidence regarding what effect dust in foreground damped systems might have on removing these lines of sight from magnitude limited quasar samples [36,9] Though the effect at high redshift is not expected to be large, it could have an important impact in the redshift range $1<z<2$. The final word on the nature of damped systems still remains to be determined.

\section{FUTURE WORK}

The future holds the potential for substantial new advances in our understanding of the evolution of galaxies using quasar absorption lines due to a number of advances. Large sky surveys, the Sloan Digital Sky Survey (e.g. [37]) and the Two Degree Field (2dF, e.g. [38]), will find substantial numbers of new quasars at low redshifts, as well as the first with redshifts $z>5$. New surveys for bright quasars $(\mathrm{R}<19.5)$ with redshifts $4<\mathrm{z}<5$ are finding many new objects for detailed follow-up of absorption systems $[39,40]$. More realistic star formation scenarios and ever faster computer will allow us to do more detailed an realistic simulations of galaxy formation to compare with observations (see [41] for a review of simulations of cosmic structure formation.) Identifying directly the galaxies responsible for damped Ly $\alpha$ absorbers has proved difficult (e.g. [42]) though progress is now being made (e.g. [43-45]. Narrow band $\mathrm{H} \alpha$ searches tuned for a damped absorber redshift may also help answer these questions (Bechtold et al. 1998).

\section{REFERENCES}

1. Rauch, M. 1998, ARA\&A, 36, 267 
2. Sargent, W.L.W., Steidel, C.C., Boksenberg, A. 1989, ApJS, 79, 703

3. Lanzetta, K.M., 1991, ApJ, 375, 1

4. Storrie-Lombardi, L.J., McMahon, R.G., Irwin, M.J., \& Hazard C. 1994, ApJ, 427, L13

5. Stengler-Larrea, E.A., Boksenberg, A., Steidel, C.C., Sargent W.L.W., Bahcall, J.N., Bergeron, J., Hartig, G.F., Januzzi, B.T., Kirhakos, S., Savage, B.D., Schneider, D.P., Turnshek, D.A. \& Weymann, R.J. 1995, ApJ, 444, 64

6. Steidel, C.C., Dickinson, M. \& Persson, S.E. 1994, ApJ, 437, L75

7. Dickinson \& Steidel 1995, BAAS, 186, 2509

8. Wolfe, A.M., Lanzetta, K.M., Foltz C.B., \& Chaffee F.H., 1995, ApJ, 454, 698

9. Pei, Y.C. \& Fall, S.M. 1995, ApJ, 454, 69

10. Lu, L., Sargent, W.L.W., Womble, D.S. \& Barlow, T.A. 1996, ApJ, 457, L1

11. Pettini, M., Smith, L.J., King, D.L., Hunstead, R.W. 1997, ApJ, 486, 665

12. Storrie-Lombardi, L.J., McMahon, R.G., Irwin, M.J., \& Hazard C. 1996, ApJ, 468, 128

13. Bahcall J.N., Peebles P.J.E. 1969, ApJ, 156, L7

14. Sargent, W.L.W., Young, P.T., Boksenberg, A. \& Tytler, D. 1980, ApJS, 42, 41

15. Lu, L., Wolfe, A.M. \& Turnshek, D.A. 1991, ApJ, 367, 19

16. Bechtold, J. 1994, ApJS, 91, 1

17. Williger, G.M., Baldwin, J.A., Carswell, R.F., Cooke, A.J., Hazard, C., Irwin, M.J., McMahon, R.G. \& Storrie-Lombardi, L.J. 1994, ApJ, 428, 574

18. Cooke, A.J., Espey, B. \& Carswell, R.F. 1997, MNRAS, 284, 552

19. Kim, T.-S., Hu, E.M., Cowie, L.L. \& Songaila, A. 1997, AJ, 114, 1

20. Press, W.H., Rybicki, G.B. \& Schneider, D.P. 1993 ApJ, 414, 64

21. Zuo, L. \& Lu, L. 1993, ApJ, 418, 601

22. Fardal, M.A., Giroux, M.L. \& Shull, J.M. 1998, AJ, 115, 2206

23. Weymann, R.J., Januzzi, B.T., Lu, L., Bahcall, J.N., Bergeron, J., Boksenberg, A., Hartig, G.F., Kirhakos, S., Sargent, W.L.W., Savage, B.D., Schneider, D.P., Turnshek, D.A. \& Wolfe, A.M. 1998, ApJ, 506, 1

24. Riediger, R., Petitjean, P. \& Mucket, J. 1998, A\&A, 329, 30

25. Storrie-Lombardi, L.J., Irwin, M.J. \& McMahon, R.G. 1996, MNRAS, 282, 1330

26. Storrie-Lombardi, L.J. \& Wolfe, A.M. 1999, in preparation

27. Vogt, S.S., Allen, S.L., Bigelow, B.C., Bresee, L., Brown, B., Cantrall, T., Conrad, A., Couture, M., Delaney, C., Epps, H.W., Hilyard, D., Hilyard, D.F.; Horn, E., Jern, N., Kanto, D., Keane, M.J., Kibrick, R.I., Lewis, J.W., Osborne, J., Pardeilhan, G.H., Pfister, T., Ricketts, T., Robinson, L.B., Stover, R.J., Tucker, D., WARD, J., \& Wei, M.Z. 1994, in proceedings SPIE, eds. Crawford, D.L. \& Craine, E.R., 2198, 362

28. Cowie, L.L., Songaila, A. Kim, T.-S. \& Hu, E.M. 1995, AJ, 109, 1522

29. Songaila, A. \& Cowie, L.L. 1996, AJ, 112, 335

30. Pettini, M., Smith, L.J., Hunstead, R.W. \& King, D.L. 1994, ApJ, 426, 79

31. Pettini, M., Ellison, S.L., Steidel, C.C. \& Bowen, D.V. 1999, ApJ, in press

32. Wolfe, A.M., Fan, X.-M., Tytler, D., Vogt, S.S., Keane, M.J. \& Lanzetta, K.M. 1994, ApJ, 435, 101

33. Prochaska, J.X. \& Wolfe, A.M. 1997a, ApJ, 474, 140 
34. Prochaska, J.X. \& Wolfe, A.M. 1997b, ApJ, 487, 73

35. Haehnelt, M.G., Steinmetz, M. \& Rauch, M. 1998, ApJ, 495,647

36. Fall, S.M. \& Pei, Y.C. 1993, ApJ, 402, 479

37. Margon, B. 1998, Philosophical Transactions of the Royal Society of London A, in press

38. Croom, S.M., Shanks, T., Boyle, B.J., Smith, R.J., Miller, L., Loaring, N.S., 1999, in proceedings of "Evolution of Large Scale Structure", in press

39. Kennefick, J.D., de Carvalho, R.R., Djorgovski, S.G., Wilber, M.M., Dickson, E.S., Weir, N., Fayyad, U. \& Roden, J., 1995, AJ, 110, 78

40. Storrie-Lombardi, L.J., Hook, I.M., Irwin, M.J. \& McMahon, R.G. 1999, in preparation

41. Weinberg, D.H., Katz, N., \& Hernquist, L. 1998, in Proceedings of 'Origins', eds. Woodward, C.E., Shull, M. \& Thronson, H. A., (Astronomical Society of the Pacific:San Francisco), Vol. 148, 47

42. Lowenthal, J.D., Hogan, C.J., Green, R.F., Woodgate, B., Caulet, A., Brown, L. \& Bechtold, J. 1995, ApJ, 451, 484

43. Djorgovski, S.G., Pahre, M.A., Bechtold, J. \& Elston, R. 1996, Nature, 382234

44. Le Brun, V., Bergeron, J., Boisse, P. \& Deharveng, J.M. 1997, A\&A, 321,733

45. Moller, P. \& Warren, S.J. 1998, MNRAS, 299, 661

46. Bechtold, J., Elston, R., Yee, H.K.C., Ellingson, E., \& Cutri, R.M. 1998, in "The Young Universe: Galaxy Formation and Evolution at Intermediate and High Redshift", eds. S. D'Odorico, A. Fontana, and E. Giallongo, ASP Conference Series, 146, 241 